\title{
RESOLVING THE BRIGHT HCN(1-0) EMISSION TOWARD THE SEYFERT 2 NUCLEUS OF M51: SHOCK ENHANCEMENT BY RADIO JETS AND WEAK MASING BY INFRARED PUMPING?
}

\author{
Satoki Matsushita $^{1}$, Dinh-V- Trung ${ }^{2}$, Frédéric Boone $^{3,4}$, Melanie Krips $^{5}$, Jeremy Lim $^{6}$, And Sebastien Muller ${ }^{7}$ \\ ${ }^{1}$ Academia Sinica Institute of Astronomy and Astrophysics, P.O. Box 23-141, Taipei 10617, Taiwan, Republic of China \\ ${ }^{2}$ Institute of Physics, Vietnamese Academy of Science \& Technology, 10, Daotan, BaDinh, Hanoi, Vietnam \\ ${ }^{3}$ Université de Toulouse, UPS-OMP, IRAP, F-31400 Toulouse, France \\ ${ }^{4}$ CNRS, IRAP, 9 Av. Colonel Roche, BP 44346, F-31028 Toulouse Cedex 4, France \\ ${ }^{5}$ Institute de Radio Astronomie Millimétrique, 300 Rue de la Piscine, F-38406 Saint Martin d'Hères, France \\ ${ }^{6}$ Department of Physics, University of Hong Kong, Pokfulam Road, Hong Kong \\ ${ }^{7}$ Department of Earth and Space Sciences, Chalmers University of Technology, Onsala Space Observatory, SE-43992 Onsala, Sweden \\ Received 2014 February 17; accepted 2014 October 27; published 2015 January 13
}

\begin{abstract}
We present high angular resolution observations of the $\mathrm{HCN}(1-0)$ emission (at $\sim 1^{\prime \prime}$ or $\sim 34 \mathrm{pc}$ ), together with $\mathrm{CO}$ $J=1-0,2-1$, and 3-2 observations, toward the Seyfert 2 nucleus of M51 (NGC 5194). The overall HCN(1-0) distribution and kinematics are very similar to that of the CO lines, which have been indicated as the jet-entrained molecular gas in our past observations. In addition, high $\mathrm{HCN}(1-0) / \mathrm{CO}(1-0)$ brightness temperature ratio of about unity is observed along the jets, similar to that observed at the shocked molecular gas in our Galaxy. These results strongly indicate that both diffuse and dense gases are entrained by the jets and outflowing from the active galactic nucleus. The channel map of $\mathrm{HCN}(1-0)$ at the systemic velocity shows a strong emission right at the nucleus, where no obvious emission has been detected in the $\mathrm{CO}$ lines. The $\mathrm{HCN}(1-0) / \mathrm{CO}(1-0)$ brightness temperature ratio at this region reaches $>2$, a value that cannot be explained considering standard physical/chemical conditions. Based on our calculations, we suggest infrared pumping and possibly weak HCN masing, but still requiring an enhanced $\mathrm{HCN}$ abundance for the cause of this high ratio. This suggests the presence of a compact dense obscuring molecular gas in front of the nucleus of M51, which remains unresolved at our $\sim 1^{\prime \prime}(\sim 34 \mathrm{pc})$ resolution, and consistent with the Seyfert 2 classification picture.
\end{abstract}

Key words: galaxies: individual (M51, NGC 5194) - galaxies: ISM - galaxies: jets - galaxies: nuclei galaxies: Seyfert - ISM: jets and outflows

\section{INTRODUCTION}

Active galactic nuclei (AGNs) are generally believed to consist of supermassive black holes surrounded by dense material (i.e., molecular gas and dust) in a disk or a torus configuration (e.g., Antonucci 1993). At spatial scales of $>100 \mathrm{pc}$, a number of observations toward nearby AGN host galaxies in molecular gas have uncovered strong concentrations of dense molecular gas at the location of AGNs (e.g., Kohno et al. 2001, 2008a), and these nuclear components often show smooth velocity gradients, suggestive of rotating disks or tori around AGNs (e.g., Jackson et al. 1993; Kohno et al. 1996; Hsieh et al. 2008; Hicks et al. 2009; Sani et al. 2012).

At finer spatial scales of $\sim 10 \mathrm{pc}$, however, molecular gas observations toward some of the previously observed AGNs revealed very different images from the past results observed at larger spatial scales. The nuclear molecular gas shows very disturbed features, such as jet-entrained or outflowing motions from AGNs (Matsushita et al. 2007; Krips et al. 2011; Combes et al. 2013; García-Burillo et al. 2014) or infalling motions toward AGNs (Davies et al. 2009; Müller Sánchez et al. 2009), with little evidence of obscuring rotating disks or tori at this spatial scale. Where is the obscuring material? In this paper, we target one of the nearest Seyfert galaxies, M51 (NGC 5194), to tackle these questions.

M51 hosts a Seyfert 2 nucleus (Ho et al. 1997) with a pair of radio jets (Ford et al. 1985; Crane \& van der Hulst 1992). It is located at a distance of 7.1 Mpc (Takáts \& Vinkó 2006), which is about one half that of NGC 1068 (14.4 Mpc; Tully 1988), and therefore a very suitable target to observe nuclear molecular gas that requires very high linear resolution. The molecular gas around the nucleus of M51 is dense $\left(>10^{4} \mathrm{~cm}^{-3}\right.$; Kohno et al. 1996) and warm (>100 K; Matsushita et al. 1998, 2004). The molecular gas distribution and kinematics within $\sim 1^{\prime \prime}(\sim 34 \mathrm{pc})$ from the AGN are complex, showing no clear evidence for a disk or torus, but instead two separated features located at the eastern and western sides of the nucleus (Matsushita et al. 2007). The western clump shows a velocity gradient along the radio jets, and its value matches well with that of the ionized gas along the jets (Bradley et al. 2004), strongly indicating that the western clump is entrained by the jets and outflowing from the nucleus (Matsushita et al. 2007).

These previous $\sim 1^{\prime \prime}$ resolution studies, however, have only been done with $\mathrm{CO}$ lines, which might trace different gas components, including diffuse molecular gas, and may confuse our view of the gas distribution and kinematics close to the nucleus. On the other hand, previous $\sim 4^{\prime \prime}$ resolution $\mathrm{HCN}(1-0)$ image (Kohno et al. 1996, 2008b) is not high resolution enough to resolve the detailed structures that have been resolved with the $\sim 1^{\prime \prime}$ CO observations. In addition, Aalto et al. (2012) reported an $\mathrm{HCN}(1-0)$ emission enhancement in the molecular outflows of Mrk 231 at a scale of about $1 \mathrm{kpc}$, suggesting that the HCN emission can be a good tool for tracing outflows. Observations toward the nucleus of M51 using the $\mathrm{HCN}(1-0)$ line at $1^{\prime \prime}$ or better resolution are therefore essential to understand the physical and chemical interactions between molecular gas and AGN jets. Here, we present $\sim 1^{\prime \prime}$ resolution $\operatorname{HCN}(1-0)$, $\mathrm{HCO}^{+}(1-0)$, and $\mathrm{HNC}(1-0)$ data toward the nuclear region of M51, together with the $\lesssim 1^{\prime \prime}$ resolution CO (1-0), (2-1), and (3-2) data, and compare the distributions and kinematics of 
these lines. We then discuss the possible emission mechanism of the HCN line from the nuclear region of M51.

\section{OBSERVATION AND DATA REDUCTION}

\subsection{PdBI Observations}

We observed the $\mathrm{HCN}(1-0), \mathrm{HCO}^{+}(1-0)$, and $\mathrm{HNC}(1-0)$ lines simultaneously toward the nuclear region of M51 using the IRAM Plateau de Bure Interferometer (PdBI). The array was in the A configuration, and the observation was carried out on 2009 February 1. The system temperatures in the single side band were in the range 80-400 K, depending mostly on the elevation angle (i.e., atmospheric absorption). Three $160 \mathrm{MHz}$ ( 535 $\mathrm{km} \mathrm{s}^{-1}$ ) bandwidth correlators were configured to cover each line. Five $320 \mathrm{MHz}$ units were configured primarily to cover nearby line-free frequencies for continuum observations and calibrations. MWC 349 was observed for the flux calibration, the strong quasar 3C273 was used for the bandpass calibration, and the quasars $\mathrm{J} 1310+323$ and $\mathrm{J} 1419+543$ were used for the phase and amplitude calibrations. The uncertainty in the absolute flux scale is estimated to be around $5 \%$.

The data were calibrated using GILDAS, and were imaged using AIPS. We did not detect any significant emission for the $\mathrm{HCO}^{+}(1-0)$ and $\mathrm{HNC}(1-0)$ lines with the $3 \sigma$ upper limit of $2.0 \mathrm{mJy}$ beam $^{-1}$ in each $20 \mathrm{~km} \mathrm{~s}^{-1}$ channel for both lines within the velocity range of $350-550 \mathrm{~km} \mathrm{~s}^{-1}$. We also did not detect any continuum emission at $90 \mathrm{GHz}$ with the $3 \sigma$ upper limit of $0.87 \mathrm{mJy}_{\text {beam }}{ }^{-1}$.

\subsection{SMA Observations}

The $\mathrm{CO}(3-2)$ line was observed with the Submillimeter Array (SMA; Ho et al. 2004) ${ }^{8}$ The observation was performed on 2005 April 18, and six out of eight $6 \mathrm{~m}$ antennas were used in the extended configuration. The correlator was configured to a $2 \mathrm{GHz}$ bandwidth with a $0.8125 \mathrm{MHz}$ channel resolution, and the line was located at the upper side band. Titan was observed as a flux calibrator, and Uranus, Ganymede, 3C279, and 3C 345 were used as bandpass calibrators. J1153+495 and 3C345 were observed every $\sim 20$ minutes for the phase and amplitude calibrations. The uncertainty in the absolute flux scale is estimated to be around $15 \%$.

The data calibrations were done using the software package MIR, adapted for $\mathrm{SMA}^{9}$ This extended configuration data set was combined with the previous compact configuration data set (Matsushita et al. 2004) in the $u v$ plane. The combined data were CLEANed and the images were produced using AIPS.

\subsection{Matching uv Ranges}

In this paper, we compare the newly obtained $\mathrm{HCN}(1-0)$ and $\mathrm{CO}(3-2)$ data with the previously obtained PdBI A configuration $\mathrm{CO}(1-0)$ data (Matsushita et al. 2007) in various ways, including line ratios. For this purpose, we need to match the shortest $u v$ lengths and the beam sizes of all the data sets, so that they all sample the same structures (i.e., the same spatial frequency components obtained by interferometers). Note that matching the beam size has the same effect as matching the longest $u v$ lengths. The shortest $u v$ length common to all data sets is

\footnotetext{
8 The Submillimeter Array is a joint project between the Smithsonian Astrophysical Observatory and the Academia Sinica Institute of Astronomy and Astrophysics, and is funded by the Smithsonian Institution and the Academia Sinica.

9 http://www.cfa.harvard.edu/ cqi/mircook.html
}

imposed by the $\mathrm{CO}(1-0)$ data, for which the shortest $u v$ length is $37 \mathrm{k} \lambda$ (i.e., we can image the structure smaller than $\sim 6^{\prime \prime}$ ). We then convolved all the images to the largest beam size among our data of $1^{\prime \prime} .10 \times 0.85$ with the position angle (P.A.) of $31^{\circ}$. We note here that this $u v$ range matching does not significantly change the images from the $u v$ unlimited ones. We did not match the $u v$ range for the $\mathrm{CO}(2-1)$ data, since there is a small overlap in the $u v$ range with other data (i.e., $\mathrm{CO} J=2-1$ data have the shortest $u v$ length twice longer than that of $J=1-0$ data, since those data are taken simultaneously with PdBI). As we will show, the $\mathrm{CO}(2-1)$ line traces the same features, and is shown for reference, although it is not included in the line ratio calculations.

\section{RESULTS}

In Figure 1, we show the central $\pm 3^{\prime \prime}( \pm 100 \mathrm{pc})$ region integrated intensity maps of the $\mathrm{CO}(1-0), \mathrm{CO}(2-1), \mathrm{CO}(3-2)$, and $\mathrm{HCN}(1-0)$ lines. All the data except $\mathrm{CO}(2-1)$ are $u v$ and beam matched images as mentioned above. The spatial resolution in $\mathrm{CO}(2-1)$ is more than a factor of two higher than other images (Matsushita et al. 2007), and is shown here for reference. All the images appear to have similar overall molecular gas distributions and kinematics. There are two clumps located at the western and eastern sides of the nucleus (hereafter clumps S1 and S2, respectively; see Figure 1(a)), whereby western clumps S1 are elongated along the northsouth direction. The intensity-weighted velocity maps for all the lines are shown in Figure 2. In all the maps, eastern clump S2 shows increasingly higher blueshifted velocities toward the nucleus, and the western clump S1 shows clear velocity gradient in the north-south direction, as was previously reported by Matsushita et al. (2007). The similarity in the kinematics for all the lines suggests that the overall molecular gas distribution and kinematics at the central $\pm 3^{\prime \prime}$ does not depend on the molecular species (both $\mathrm{CO}$ and $\mathrm{HCN}$ ) or properties (both diffuse and dense gas, and from lower- $J$ to higher- $J$ lines). As mentioned in Section 1, the S1 clump is the jet-entrained molecular gas, so that the similarity of the distribution and kinematics between the $\mathrm{CO}$ and $\mathrm{HCN}$ data strongly indicates that the both diffuse and dense molecular gas is entrained by the jets and outflowing from the AGN.

With a detailed inspection of the channel maps, however, a distinct difference between the $\mathrm{CO}$ and $\mathrm{HCN}$ distributions can be seen in a channel map at the systemic velocity (471.7 \pm $0.3 \mathrm{~km} \mathrm{~s}^{-1}$ Shetty et al. 2007). Figure 3 displays three channel maps centered at the velocity of 460,480 , and $500 \mathrm{~km} \mathrm{~s}^{-1}$, each with a width of $\sim 20 \mathrm{~km} \mathrm{~s}^{-1}$. In the channel maps at the velocity of 460 and $500 \mathrm{~km} \mathrm{~s}^{-1}$ (i.e., adjacent to the channel at the systemic velocity), all the lines show the emission peaks at either or both eastern and western sides of the nucleus (some emissions elongate toward the nucleus, but do not peak there). On the other hand, in the channel maps at the velocity of $480 \mathrm{~km} \mathrm{~s}^{-1}$, which includes the systemic velocity of $471.7 \pm 0.3 \mathrm{~km} \mathrm{~s}^{-1}$, the $\mathrm{HCN}(1-0)$ line clearly exhibits a $\sim 5 \sigma$ peak at the nucleus, where $\mathrm{CO}$ lines show no peak if any detectable emission at the nucleus. To further confirm this systemic velocity $\mathrm{HCN}$ emission peak, we plot the spectra toward the nucleus of M51 for all four lines in Figure 4. As can be seen, all the CO lines exhibit at best emission at the $\sim 2 \sigma$ level, but the $\mathrm{HCN}(1-0)$ line shows a $\sim 5 \sigma$ peak only at the systemic velocity channel of $480 \mathrm{~km} \mathrm{~s}^{-1}$. This therefore indicates that the central $\mathrm{HCN}(1-0)$ emission is highly related to the molecular gas around the systemic velocity. 

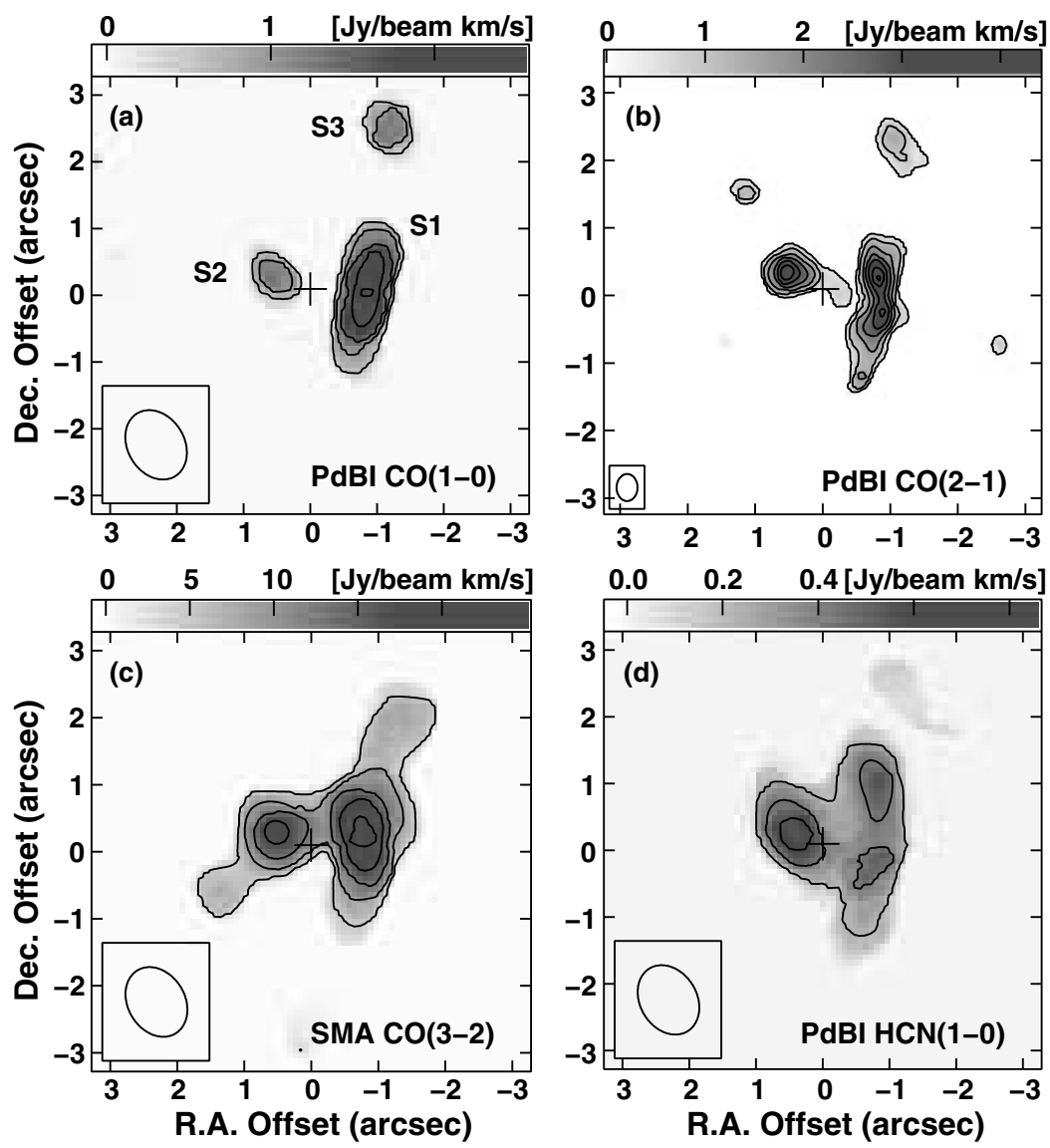

Figure 1. Integrated intensity maps of (a) $\mathrm{CO}(1-0)$, (b) $\mathrm{CO}(2-1)$, (c) $\mathrm{CO}(3-2)$, and (d) $\mathrm{HCN}(1-0)$ lines toward the central $\pm 3^{\prime \prime}$ ( $\pm 100 \mathrm{pc}$ ) of $\mathrm{M} 51$ (the $\mathrm{CO} J=2-1$ image is from Matsushita et al. 2007). The $u v$ coverages and the beam sizes $\left(1^{\prime \prime} 10 \times 00^{\prime \prime} 85\right.$, P.A. $\left.=31^{\circ}\right)$ are matched, except the $\mathrm{CO}(2-1)$ line map $\left(00^{\prime \prime} 40 \times 0\right.$.' 31 , P.A. $=0^{\circ}$ ). The central cross in each map displays the location of the type 2 Seyfert nucleus determined from the peak of the $8.4 \mathrm{GHz}$ radio continuum peak position of R.A. $=13^{\mathrm{h}} 29^{\mathrm{m}} 52^{\mathrm{s}} .7101$ and decl. $=47^{\circ} 11^{\prime} 42^{\prime \prime} 696$ (Hagiwara et al. 2001; Bradley et al. 2004). In (a), three molecular gas clumps have been labeled in the same manner as in Scoville et al. (1998). Contour levels are as follows: (a) 2, 4, 6, 8, and $10 \times 0.248 \mathrm{Jy} \mathrm{beam}^{-1} \mathrm{~km} \mathrm{~s}^{-1}$; (b) 1, 3, 5, 7, 9, and $11 \times 0.334 \mathrm{Jy}$ beam ${ }^{-1} \mathrm{~km} \mathrm{~s}^{-1}$; (c) $2,4,6,8$, and $10 \times 2.21 \mathrm{Jy} \mathrm{beam}^{-1} \mathrm{~km} \mathrm{~s}^{-1}$; (d) 2,4 , and $6 \times 0.109 \mathrm{Jy} \mathrm{beam}^{-1} \mathrm{~km} \mathrm{~s}^{-1}$.

After converting the integrated intensity maps of the $\mathrm{CO}(1-0)$ and $\mathrm{HCN}(1-0)$ lines in Figure 1 into brightness temperature maps, we made the $\mathrm{HCN}(1-0) / \mathrm{CO}(1-0)$ brightness temperature ratio map in Figure 5 overlaid on the VLA $6 \mathrm{~cm}$ continuum map (Crane \& van der Hulst 1992). All the molecular gas clumps show the $\mathrm{HCN} / \mathrm{CO}$ ratios of more than 0.1 , and the clumps closer in projection either to the AGN or to the jet tend to have higher ratios. Indeed, the highest ratio of more than 2.0 is located at the AGN (western) side of clump S2. More interestingly, all the eastern side of clump S1 has higher ratio of $\sim 1.0$ than the western side of $\sim 0.3-0.5$, and this side is where the radio jets are running through, which can be clearly seen in Figure 5.

The average ratios of clumps $\mathrm{S} 1, \mathrm{~S} 2$, and $\mathrm{S} 3$ are calculated as $0.6,1.5$, and 0.3 , respectively. On the other hand, since the $\mathrm{CO}(1-0)$ emission totally lacks at the nucleus (see Figure 1(a)), the $\mathrm{HCN} / \mathrm{CO}$ ratio at the nucleus cannot be calculated in the ratio map. But since the $\mathrm{HCN}(1-0)$ emission does exist at the nucleus with the integrated intensity of $0.422 \mathrm{Jy} \mathrm{beam}^{-1} \mathrm{~km} \mathrm{~s}^{-1}$, the lower limit of the $\mathrm{HCN} / \mathrm{CO}$ brightness temperature ratio at the nucleus can be calculated as $>0.96$ using the $3 \sigma$ upper limit of CO of $0.744 \mathrm{Jy}_{\text {beam }}{ }^{-1} \mathrm{~km} \mathrm{~s}^{-1}$. This is also true in the channel map at the velocity of $480 \mathrm{~km} \mathrm{~s}^{-1}$; in Figure 3 , no clear emission in $\mathrm{CO}(1-0)$ toward the nucleus with $1 \sigma$ noise level of $0.45 \mathrm{~K}$, while $\mathrm{HCN}(1-0)$ is detected with $1.5 \mathrm{~K}(\sim 5 \sigma)$.

The radial tendency of the $\mathrm{HCN} / \mathrm{CO}$ ratio is more clearly seen in the radial ratio plot. Figure 6(a) plots the radial integrated intensity distributions of $\mathrm{HCN}(1-0)$ and $\mathrm{CO}(1-0)$ in units of $\mathrm{K}$ $\mathrm{km} \mathrm{s}^{-1}$. It is obvious that the $\operatorname{HCN}(1-0)$ emission is centrally concentrated, while the $\mathrm{CO}(1-0)$ emission exhibits deficiency at the nucleus. From these two radial distributions, we made the radial $\mathrm{HCN}(1-0) / \mathrm{CO}(1-0)$ integrated intensity ratio plot in Figure 6(b). This figure clearly displays that the $\mathrm{HCN} / \mathrm{CO}$ ratio is higher at the nucleus and decreases monotonically as the distance from the nucleus increases out to $\sim 1^{\prime \prime} .25$.

As mentioned in Section 2.1, we did not detect the $\mathrm{HCO}^{+}(1-0)$ and $\mathrm{HNC}(1-0)$ lines and therefore the maps for these lines are not presented in this paper. The brightness temperature ratios for both $\mathrm{HCN}(1-0) / \mathrm{HCO}^{+}(1-0)$ and $\mathrm{HCN}(1-0) / \mathrm{HNC}(1-0)$ are therefore $>4.3$ using $3 \sigma$ upper limits for these non-detected lines.

\section{DISCUSSION}

\subsection{High HCN/CO Ratios at the Nucleus and Outflowing Gas}

Our high spatial resolution molecular gas images toward the Seyfert 2 nucleus of M51 clearly exhibit higher $\operatorname{HCN}(1-0)$ / $\mathrm{CO}(1-0)$ ratios in the molecular gas clumps located close to the AGN than those in other galaxies; the jet-entrained molecular gas clump (clump $\mathrm{S} 1$; projected distance of $\sim 30 \mathrm{pc}$ from the nucleus) has an averaged $\mathrm{HCN} / \mathrm{CO}$ ratio of 0.6 , and the nearest clump from the AGN (clump S2; projected distance $<20 \mathrm{pc}$ ) a 

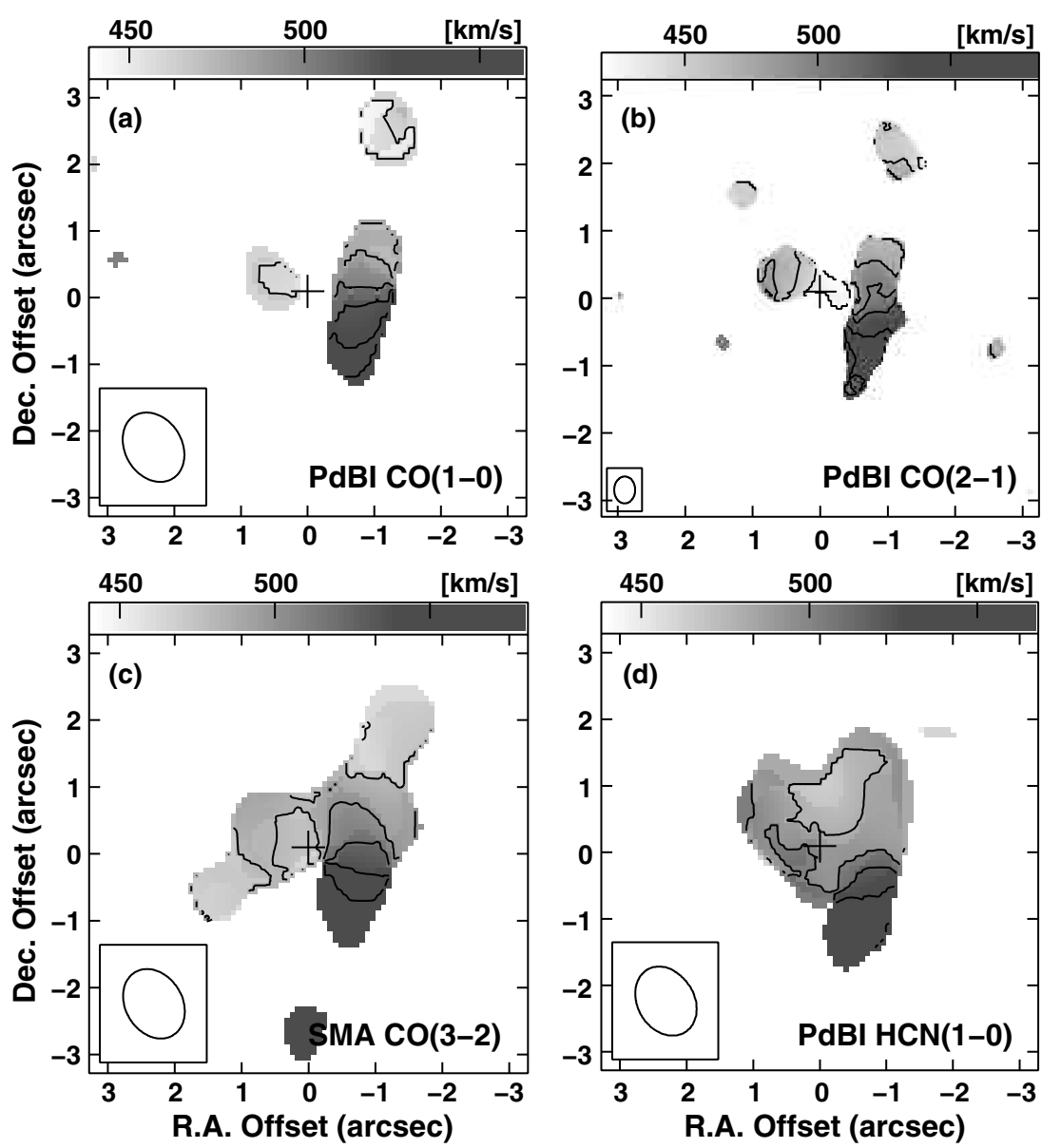

Figure 2. Intensity-weighted velocity field maps of (a) $\mathrm{CO}(1-0)$, (b) $\mathrm{CO}(2-1)$, (c) $\mathrm{CO}(3-2)$, and (d) $\mathrm{HCN}(1-0)$ lines toward the central $\pm 3^{\prime \prime}$ of $\mathrm{M} 51$ (the $\mathrm{CO} J=$ $2-1$ image is from Matsushita et al. 2007). Contour levels are as follows: (a) $460,480,500, \ldots, 560 \mathrm{~km} \mathrm{~s}^{-1}$; (b) $440,460,480, \ldots, 540 \mathrm{~km} \mathrm{~s}^{-1}$; (c) $460,480,500, \ldots$, $560 \mathrm{~km} \mathrm{~s}^{-1}$; (d) $480,500,520,540$, and $560 \mathrm{~km} \mathrm{~s}^{-1}$. Other information is the same as in Figure 1.

ratio of 1.5. Clump $\mathrm{S} 3$, which is located farthest from the AGN, has a ratio of 0.3 .

In normal and starburst galaxies, the $\mathrm{HCN}(1-0) / \mathrm{CO}(1-0)$ ratios are < 0.3 (e.g., Solomon et al. 1992; Helfer \& Blitz 1993; Aalto et al. 1995; Sorai et al. 2002; Gao \& Solomon 2004; Kohno et al. 2008a; Matsushita et al. 2010). The ratios in our Galaxy and M31 are also low, around 0.01-0.03 for Giant Molecular Clouds (GMCs) in the galactic disks (Helfer \& Blitz 1997; Brouillet et al. 2005), and up to $\sim 0.15$ at the Galactic center (Jackson et al. 1996). The ratios can be up to $\sim 0.6$ for the case of molecular gas located around Seyfert nuclei (e.g., Kohno et al. 2008a; Krips et al. 2007, 2011). Based on these values, the averaged $\mathrm{HCN} / \mathrm{CO}$ ratio of clump S1 is similar to those of other Seyfert galaxies, and that of clump S3 is at the high end of normal or starburst galaxies. Clump S2 is exceptional; the $\mathrm{HCN}$ intensity is brighter than that of $\mathrm{CO}$, and this has never been observed in other galaxies in the past.

\subsection{Shocked Molecular Gas Outflow along the Radio Jets}

As shown in Section 3 and in Figure 5, the S1 clump shows a high $\mathrm{HCN} / \mathrm{CO}$ ratio of around unity only at the side where the radio jets overlap. In addition, this clump is known to be an outflow that is entrained by the radio jets (Matsushita et al. 2007). Such a high ratio has been observed at shocked regions of molecular outflows from young stellar objects in our Galaxy.

Umemoto et al. (1992) observed a highly collimated outflow in the dark cloud $\mathrm{L} 1157$ with the ${ }^{12} \mathrm{CO}(1-0),{ }^{13} \mathrm{CO}(1-0)$, and
$\mathrm{HCN}(1-0)$ lines. In their ${ }^{12} \mathrm{CO}$ spectrum, two (narrow and broad) components can be seen, which are tracing quiescent and outflowing gas, respectively. In the ${ }^{13} \mathrm{CO}$ spectrum, only a narrow component has been detected, but on the contrary, only a broad component has been detected in the HCN spectrum. Using their data, we derive the line ratios with assuming that the $\mathrm{HCN}$ emission is only from the outflow component and the ${ }^{13} \mathrm{CO}$ emission from quiescent component. For the ${ }^{12} \mathrm{CO}$ line, we fit a Gaussian to each component to derive the integrated intensity. We integrate all the hyperfine components of the HCN line, since our M51 data cannot distinguish these lines. From these data, we calculated the $\mathrm{HCN} /{ }^{12} \mathrm{CO}$ and $\mathrm{HCN} /{ }^{13} \mathrm{CO}$ ratios for the outflowing (shocked) gas as 0.9 and $>260$ (using $3 \sigma$ upper limit for the ${ }^{13} \mathrm{CO}$ data), respectively. Interferometric $\mathrm{HCN}$ and ${ }^{12} \mathrm{CO}$ line observations of the Orion-KL high velocity outflow (Wright et al. 1996) also show high $\mathrm{HCN} /{ }^{12} \mathrm{CO}$ ratios ranging between $0.4-0.5$. The ratios in the Orion-KL outflow is lower than that in the L1157 outflow, possibly due to more complex nature (and therefore larger contamination) in the massive star forming regions than in the low mass star forming regions.

The ratio and the condition of clump S1, especially the side close to the radio jets, is very similar to that of the outflowing (shocked) molecular gas in L1157. This strongly supports the idea that the high $\mathrm{HCN} / \mathrm{CO}$ ratio along the radio jets in M51 is caused by the strong interaction (i.e., shock) between the jets and the outflowing molecular gas. Furthermore, it is worth mentioning that the averaged $\mathrm{HCN} / \mathrm{CO}$ ratio of 0.6 is very 

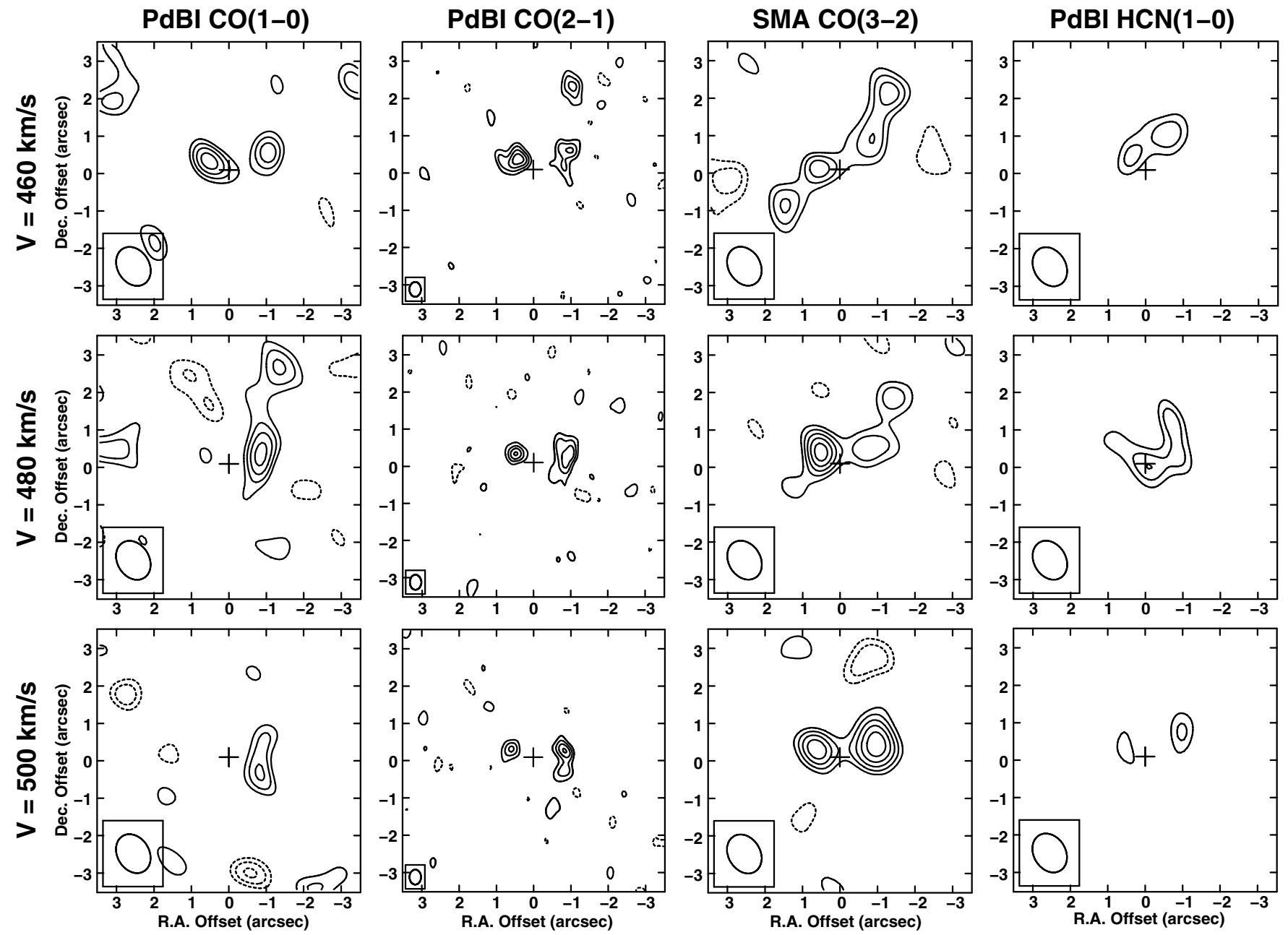

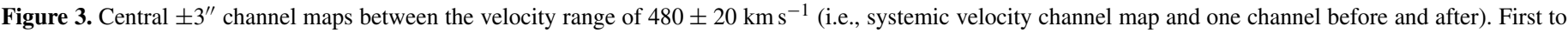

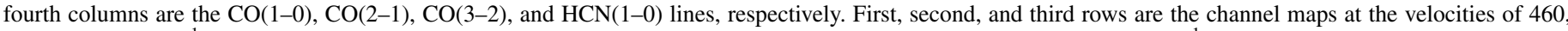

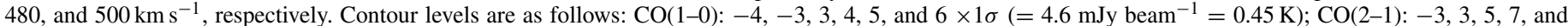

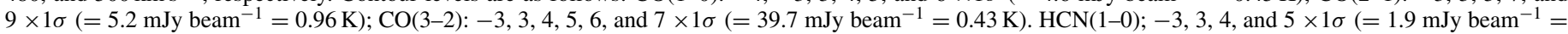
$0.31 \mathrm{~K})$. Note that there was no positive or negative noise more than $3 \sigma$ in the HCN(1-0) map. See the Figure 1 caption for other information.

similar to the dense molecular gas outflow observed toward Mrk 231 of $\sim 0.6$ (Aalto et al. 2012). Since their spatial resolution of $\sim 1.2 \mathrm{kpc}$ is lower than ours, it is possible that the actual $\mathrm{HCN} / \mathrm{CO}$ ratio in Mrk 231 is higher.

\subsection{Infrared Pumping with Weak Maser at Circumnuclear Molecular Gas?}

Clump S2 shows an extremely high $\mathrm{HCN} / \mathrm{CO}$ ratio of 1.5 on average, and more than 2 at the nucleus as shown in Figure 6. As mentioned above, such high ratios have not been observed in extragalactic molecular gas, but observed in molecular gas around asymptotic giant branch (AGB) stars.

We observed the high mass-loss carbon AGB stars, IRC+10216, with ${ }^{12} \mathrm{CO}(1-0),{ }^{13} \mathrm{CO}(1-0)$, and $\mathrm{HCN}(1-0)$ for the intensity calibrations of our past observations (see Matsushita et al. 2010 for details). The resultant ${ }^{12} \mathrm{CO} /{ }^{13} \mathrm{CO}, \mathrm{HCN} /{ }^{12} \mathrm{CO}$, and $\mathrm{HCN} /{ }^{13} \mathrm{CO}$ integrated intensity ratios are $\sim 11,1.3$, and 15 , respectively. Note again that we integrate all the $\mathrm{HCN}$ hyperfine lines for this calculation. The high $\mathrm{HCN} /{ }^{12} \mathrm{CO}$ and $\mathrm{HCN} /{ }^{13} \mathrm{CO}$ ratios are due to the infrared (IR) pumping (Dinh-V-Trung \& Nguyen-Q-Rieu 2000).

This result suggests that the extremely high $\mathrm{HCN} / \mathrm{CO}$ ratio can be due to the IR pumping. In the following subsections, we discuss what is the possible excitation mechanism for clump S2.

\subsubsection{Excitation Mechanisms of the HCN Emission: I. LTE/Non-LTE Conditions}

We start by assuming that both molecules are excited solely by collisions with $\mathrm{H}_{2}$ molecules, which is typically the case in Galactic GMCs. The opacity ratio under the local thermal equilibrium (LTE) can be expressed as

$$
\begin{aligned}
\frac{\tau_{\mathrm{HCN}(1-0)}}{\tau_{\mathrm{CO}(1-0)}}= & \frac{f_{\mathrm{HCN}}}{f_{\mathrm{CO}}}\left(\frac{\mu_{\mathrm{HCN}}}{\mu_{\mathrm{CO}}}\right)^{2} \frac{B_{\mathrm{HCN}}}{B_{\mathrm{CO}}} \frac{[\mathrm{HCN}]}{[\mathrm{CO}]} \\
& \times \frac{1-\exp \left(-h \nu_{\mathrm{HCN}(1-0)} / k T\right)}{1-\exp \left(-h v_{\mathrm{CO}(1-0)} / k T\right)},
\end{aligned}
$$

where $f$ is the filling factor, $\mu$ is the dipole moment, $B$ is the rotational constant, $h$ is the Planck constant, $v$ is the rotational transition frequency, and $k$ is the Boltzmann constant. The HCN and $\mathrm{CO}$ molecular abundances relative to $\mathrm{H}_{2}$ are expressed as $[\mathrm{HCN}]$ and $[\mathrm{CO}]$, respectively. Adopting $f_{\mathrm{HCN}}=f_{\mathrm{CO}}$, which is already an extreme case compared to the Galactic GMCs where $f_{\mathrm{HCN}} \ll f_{\mathrm{CO}}, \mu_{\mathrm{HCN}}=2.984, \mu_{\mathrm{CO}}=0.112$, 

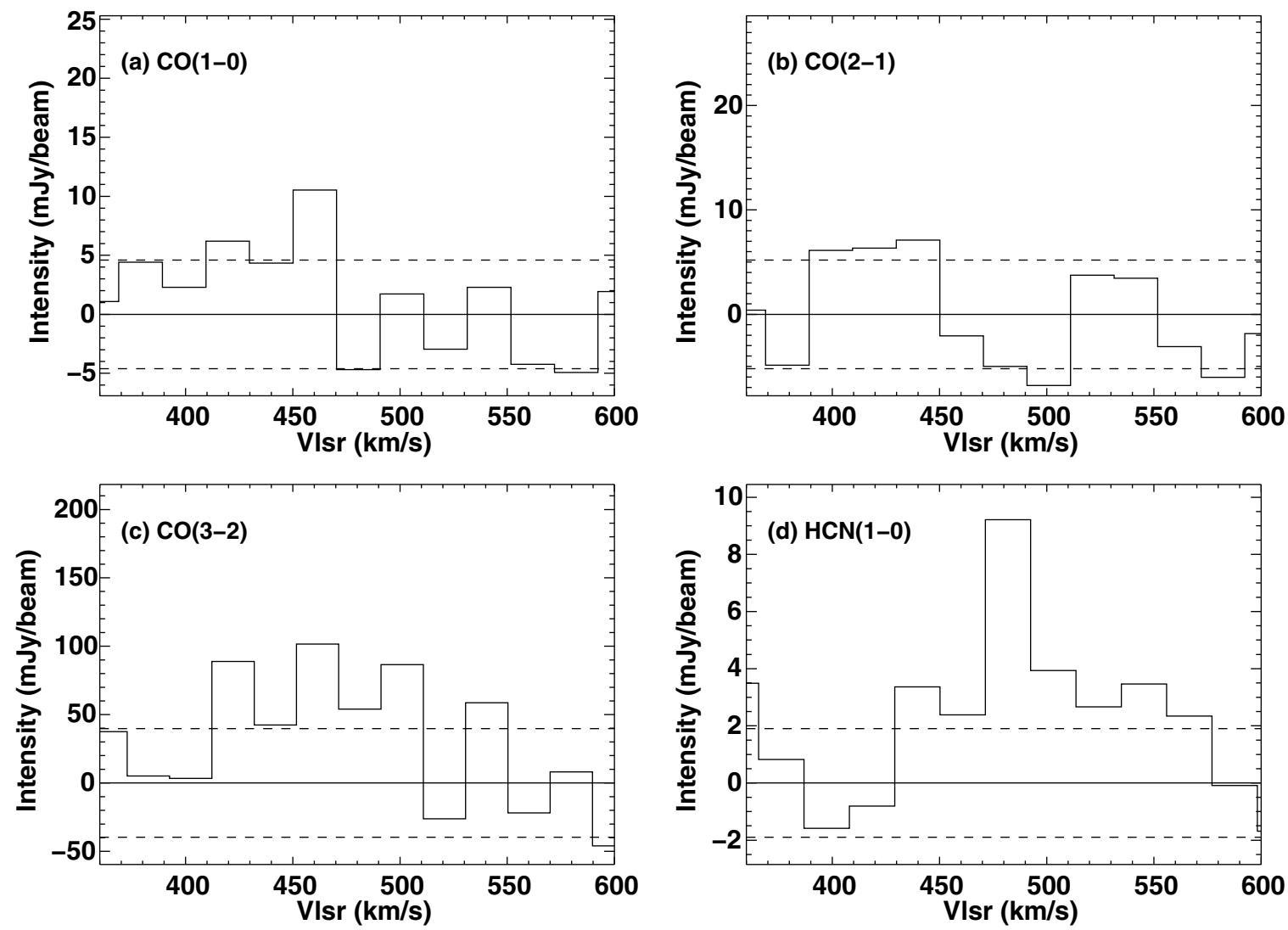

Figure 4. Spectra toward the M51 nucleus for (a) $\mathrm{CO}(1-0)$, (b) $\mathrm{CO}(2-1)$, (c) $\mathrm{CO}(3-2)$, and (d) $\mathrm{HCN}(1-0)$ lines. The spectra have been taken at the position of the nucleus with the spatial resolution the same as the beam sizes. The nuclear position and the beam sizes are mentioned in the caption of Figure 1. Horizontal dashed lines in each plot are $\pm 1 \sigma$ of each data set (see the caption of Figure 1 for the values). All the CO lines exhibit at most $\sim 2 \sigma$ peaks, but the HCN(1-0) line only at the velocity channel of $480 \mathrm{~km} \mathrm{~s}^{-1}$, which includes the systemic velocity of $471.7 \pm 0.3 \mathrm{~km} \mathrm{~s}^{-1}$ (Shetty et al. 2007), shows a $\sim 5 \sigma$ peak.

$B_{\mathrm{HCN}}=44.316 \mathrm{GHz}, \quad B_{\mathrm{CO}}=57.636 \mathrm{GHz}, \quad \nu_{\mathrm{HCN}}=$ $88.631 \mathrm{GHz}, v_{\mathrm{CO}}=115.271 \mathrm{GHz},[\mathrm{HCN}]=2 \times 10^{-8}$ (Irvine et al. 1987), and [CO] $=5 \times 10^{-5}$, the opacity ratio can be calculated as $\tau_{\mathrm{HCN}(1-0)} / \tau_{\mathrm{CO}(1-0)} \sim 0.18 \pm 0.01$ under a large range of molecular gas temperature condition $(T \sim 3-1000 \mathrm{~K})$. The brightness temperature ratio can therefore be expressed as

$$
\begin{aligned}
\frac{T_{\mathrm{HCN}(1-0)}}{T_{\mathrm{CO}(1-0)}} & =\frac{1-\exp \left(-\tau_{\mathrm{HCN}}\right)}{1-\exp \left(-\tau_{\mathrm{CO}}\right)} \\
& \sim \frac{1-\exp \left(-0.18 \tau_{\mathrm{CO}}\right)}{1-\exp \left(-\tau_{\mathrm{CO}}\right)},
\end{aligned}
$$

and this ratio ranges between 0.18 (optically thin limit) and 1 (optically thick limit), which cannot realize $\operatorname{HCN}(1-0) /$ $\mathrm{CO}(1-0)>1$.

If $f_{\mathrm{HCN}}$ is $\sim 10$ times larger than $f_{\mathrm{CO}}$, then it is possible to obtain the observed ratio, but it is physically unlikely; the critical density of the HCN molecule is more than an order of magnitude larger than that of the $\mathrm{CO}$ molecule, so that wherever the $\mathrm{HCN}$ molecules can emit, the $\mathrm{CO}$ molecules can also emit, and $f_{\mathrm{HCN}}$ cannot be larger than $f_{\mathrm{CO}}$. A simple way to reproduce the observed $\mathrm{HCN} / \mathrm{CO}$ ratio would be if the $[\mathrm{HCN}] /[\mathrm{CO}]$ abundance ratio is increased by a factor of $\sim 10$. In either case, the $\mathrm{CO}$ emission should be optically thin. This is also true even for the non-LTE radiation transfer modeling using the large-velocity-gradient (LVG) approximation; without changing the $\mathrm{HCN}$ abundance, the $\mathrm{HCN} / \mathrm{CO}$ ratio can be at most around unity under very limited temperature or density range (see Section 4.3.2 and Figure 7(c)).

\subsubsection{Excitation Mechanisms of the $\mathrm{HCN}$ Emission: II. Infrared Pumping}

In addition to the collisional excitation, here we also consider the possibility of IR radiative pumping (Morris 1975; Carrol \& Goldsmith 1981; Deguchi \& Uyemura 1984), since this mechanism can enhance the $\mathrm{HCN}$ intensity as mentioned for the case of IRC+10216 in Section 4.3. This mechanism is to pump the population of a rotational level to higher levels via vibrational transitions; in a strong IR radiation field, some molecules that have vibrational transitions at an IR wavelength will be pumped to higher vibrational levels and radiate back to lower levels, but to higher rotational levels than before. This process increases the population at higher rotational levels and therefore makes the rotational transition emission stronger than general molecular clouds (no strong IR field), which only have collisional pumping. This is particularly relevant when a luminous source is present (e.g., AGN) in the background, such that optical/UV radiation is absorbed by dust and reradiated in the IR. The vibrational transition of $\mathrm{HCN}$ molecule is at $14 \mu \mathrm{m}$, where the radiation from warm dust irradiated by AGNs is strong, and therefore this is one of the possible mechanisms to explain the high $\mathrm{HCN} / \mathrm{CO}$ ratio toward AGNs. The absorption line of the $\mathrm{HCN} 14 \mu \mathrm{m}$ transition can be seen in several IR luminous galaxies (Lahuis et al. 2007; Farrah et al. 2007). Recent interferometric observations toward those galaxies have indeed displayed vibrationally excited $\mathrm{HCN}$ lines toward their IR luminous nuclei (Salter et al. 2008; Sakamoto et al. 2010; Imanishi et al. 2013). 


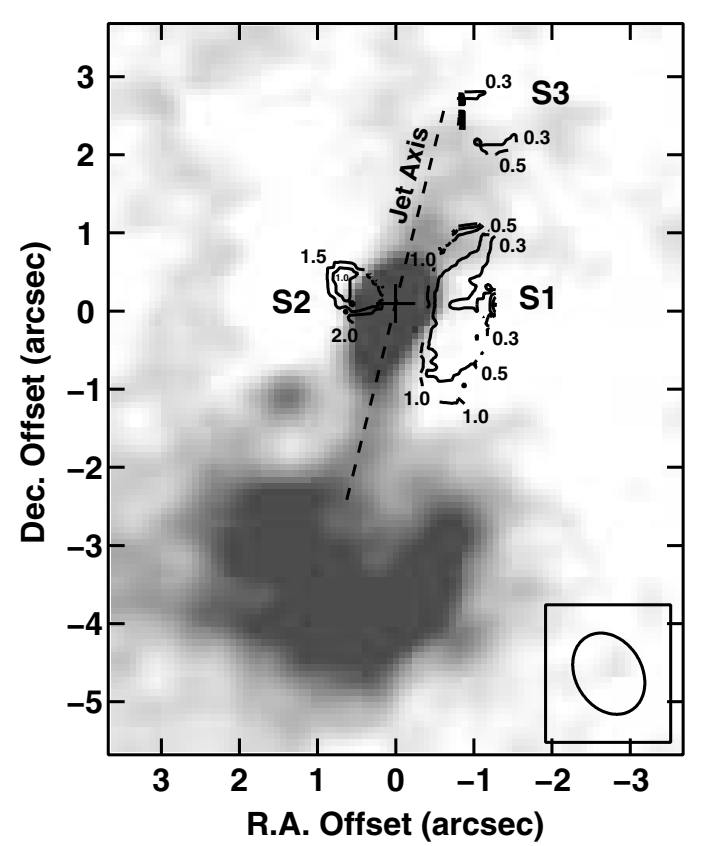

Figure 5. $\mathrm{HCN}(1-0) / \mathrm{CO}(1-0)$ brightness temperature ratio map (contours) overlaid on the VLA $6 \mathrm{~cm}$ radio continuum image (grayscale; Crane \& van der Hulst 1992). Contour levels are presented in the map. The dashed line shows the radio jet axis (P.A. $=166^{\circ}$; Bradley et al. 2004), and the continuum image is enhanced to show the weak jet emission elongated toward southeast, and connected to the bow-shock emission at southeast of this image. The average ratios for clumps $\mathrm{S} 1, \mathrm{~S} 2$, and $\mathrm{S} 3$ are calculated as $0.6,1.5$, and 0.3 , respectively. $\mathrm{S} 1$ is clearly elongated along this jet emission (see also Matsushita et al. 2007), and the higher ratio regions are concentrated where S1 and the jet overlap. S2 mostly overlaps with the jet emission and has high ratio overall, but is higher toward the Seyfert 2 nucleus. The cross at the center is the same as in Figure 1.

To check whether IR pumping of HCN can occur near the nucleus, we need to estimate the radiative excitation in this region. The condition for the IR pumping to be effective is when the probability of the $\mathrm{HCN} v=0-1$ vibrational transition absorption at $14 \mu \mathrm{m}, B_{v=0-1} I_{14 \mu \mathrm{m}}\left(\mathrm{s}^{-1}\right)$, is larger than that of the $\mathrm{HCN} J=1-0$ rotational transition spontaneous emission, $A_{J=1-0}\left(\mathrm{~s}^{-1}\right)$, namely,

$$
B_{v=0-1} I_{14 \mu \mathrm{m}} \geqslant A_{J=1-0},
$$

where $I_{14 \mu \mathrm{m}}$ is the average intensity of the radiation field at $14 \mu \mathrm{m}$. This equation can be re-written as

$$
\frac{c^{2}}{2 h \nu^{3}} A_{v=1-0} I_{14 \mu \mathrm{m}} \geqslant A_{J=1-0},
$$

where $c$ is the speed of light, $h$ is the Planck constant, and $v$ is the frequency. We use $A_{v=1-0}=2.23 \mathrm{~s}^{-1}$ (Harris et al. 2006) from the GEISA spectroscopic database (Jacquinet-Husson et al. 2011) and $A_{J=1-0}=2.41 \times 10^{-5} \mathrm{~s}^{-1}$ (Dumouchel et al. 2010) from RADEX (Van der Tak et al. 2007) for this calculation.

To estimate the radiation field at $14 \mu \mathrm{m}$ around the nucleus of M51, we use the recent high resolution images taken with the photometric bands S11 $(11 \mu \mathrm{m})$ and L15 $(15 \mu \mathrm{m})$ of the AKARI Infrared Camera (Egusa et al. 2013). Both images show a point-like source at the nucleus of M51 at a spatial resolution of 7".4, and well separated from the surrounding spiral arms (i.e., no contamination from the emission of the spiral arms; see Figure 2 of Egusa et al. 2013). We therefore assume that the point-like emission at the nucleus of M51 in these images originates from the compact Seyfert 2 nucleus. The flux densities of the nuclear emission at S11 and L15 are $3.7 \times 10^{-2} \mathrm{Jy}$ and $6.4 \times 10^{-2} \mathrm{Jy}$, respectively. With linear interpolation between S11 and L15 flux densities, we estimate a $14 \mu \mathrm{m}$ flux density, $S_{14 \mu \mathrm{m}}$, of $5.7 \times 10^{-2} \mathrm{Jy}$.

$I_{14 \mu \mathrm{m}}$ can be calculated using $S_{14 \mu \mathrm{m}}$ as

$$
I_{14 \mu \mathrm{m}}=\frac{S_{14 \mu \mathrm{m}}}{\Omega_{14 \mu \mathrm{m}}},
$$

where $\Omega_{14 \mu \mathrm{m}}$ is the solid angle of the source at $14 \mu \mathrm{m}$. Since the compact infrared nuclear source of M51 has not been resolved so far, we adopt the size of the infrared source as a few (2-3) pc, which is the same as that of the thick dust disk observed toward the nucleus of the prototypical Seyfert 2 galaxy NGC 1068 (Jaffe et al. 2004). Substituting the above values into Equations (5) and (4), the left-hand side of the Equation (4) is calculated as (3.3-9.2) $\times 10^{-5} \mathrm{~s}^{-1}$, which is larger than the right-hand side of the equation of $2.41 \times 10^{-5} \mathrm{~s}^{-1}$. This indicates that IR pumping can occur in the molecular gas surrounding the Seyfert 2 nucleus of M51.

We then included the infrared pumping effect in our LVG calculations. We use the above calculated $14 \mu \mathrm{m}$ intensity for the radiation coming from the background infrared source. Here, we adopt the infrared source size of $3 \mathrm{pc}$. We also assume that the molecular gas has a temperature of $100 \mathrm{~K}$. Note that in general higher molecular gas temperature induces more collision, and therefore decreases the effect of infrared pumping. But under the high temperature condition, the effect of infrared pumping to lower transitions is insensitive to changes in the molecular gas temperature, since most of population is at higher transitions; a factor of a few change in temperature gives almost no difference in the calculation results.

In the first calculation, we use the $\mathrm{H}_{2}$ column density per velocity of $1 \times 10^{21} \mathrm{~cm}^{-2}$, which corresponds to the $\mathrm{H}_{2}$ column density toward the nucleus of M51 $\left(6.2 \times 10^{21} \mathrm{~cm}^{-2}\right.$; Matsushita et al. 2007) divided by the typical velocity width of the extragalactic molecular clouds of 6-8 $\mathrm{km} \mathrm{s}^{-1}$ (Combes \& Becquaert 1997). We also assume that the column densities per velocity for $\mathrm{CO}$ and $\mathrm{HCN}, N_{\mathrm{CO}} / d v$ and $N_{\mathrm{HCN}} / d v$, are $5 \times 10^{16} \mathrm{~cm}^{-2}$ $\left(\mathrm{km} \mathrm{s}^{-1}\right)^{-1}$ and $3 \times 10^{13} \mathrm{~cm}^{-2}\left(\mathrm{~km} \mathrm{~s}^{-1}\right)^{-1}$, respectively. These column densities for $\mathrm{CO}$ and $\mathrm{HCN}$ correspond to the normal abundance ratio mentioned in Section 4.3.1 relative to the $\mathrm{H}_{2}$ column density mentioned above. The results for the brightness temperatures of $\mathrm{CO}$ (black curves) and HCN (red curves) lines are shown in Figure 7(a), with the comparison of the results without infrared pumping (Figure 7(c)). As can be seen, using the normal abundance of $\mathrm{HCN}$ and $\mathrm{CO}$, the $\mathrm{HCN}(1-0) / \mathrm{CO}(1-0)$ ratio of at most about unity can be realized under very limited condition of $n_{\mathrm{H}_{2}} \sim 10^{5-6} \mathrm{~cm}^{-3}$, but cannot realize $\mathrm{HCN}(1-0) /$ $\mathrm{CO}(1-0)>2$ both with and without infrared pumping.

We then repeated the calculations by increasing the $\mathrm{HCN}$ abundance by an order of magnitude, namely, changing $N_{\mathrm{HCN}} / d v$ to $3 \times 10^{14} \mathrm{~cm}^{-2}\left(\mathrm{~km} \mathrm{~s}^{-1}\right)^{-1}$. The calculation results are displayed in Figure 7(b), with the comparison of the results without infrared pumping (Figure $7(\mathrm{~d})$ ). In both cases, a high $\mathrm{HCN}(1-0) / \mathrm{CO}(1-0)$ ratio of $>2$ can be obtained at dense condition $\left(n_{\mathrm{H}_{2}}>10^{5} \mathrm{~cm}^{-3}\right)$. On the other hand, when including infrared pumping, $\mathrm{HCN}(1-0) / \mathrm{CO}(1-0)>2$ can also be obtained even at low density condition, namely, it is possible at a large density range.

Note that with the $\mathrm{H}_{2}$ column density per velocity we used, the $\mathrm{CO}$ brightness temperatures, especially $J=1-0$, are much lower than the assumed molecular gas temperature of $100 \mathrm{~K}$, 

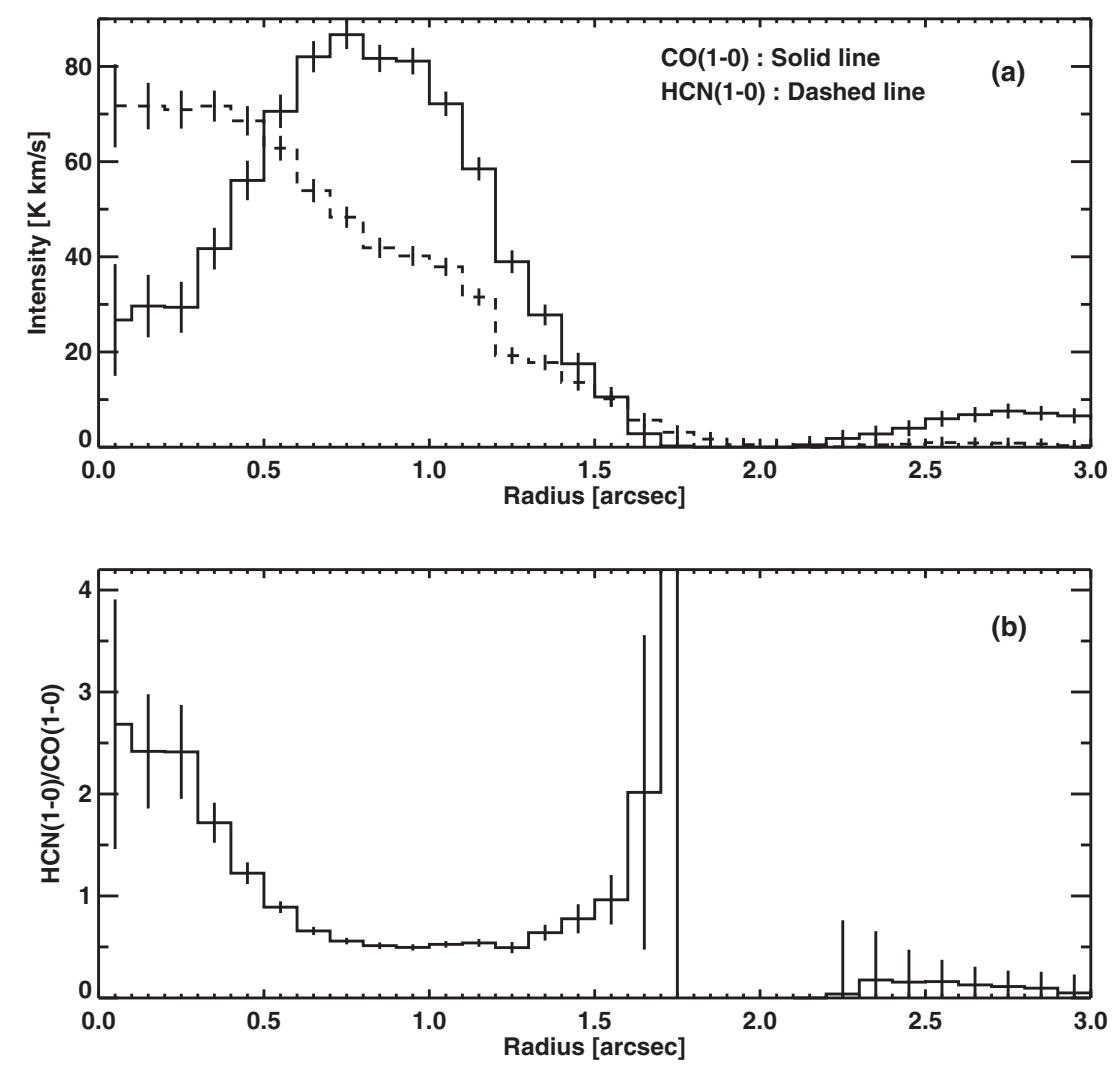

Figure 6. (a) Central $3^{\prime \prime}$ radial intensity distributions of the $\mathrm{CO}(1-0)$ (solid line) and $\mathrm{HCN}(1-0)$ (dashed line) emission derived from the integrated intensity maps in Figure 1 after converted into brightness temperature scale. (b) Central $3^{\prime \prime} \mathrm{HCN} / \mathrm{CO}$ integrated intensity ratio distribution. The $u v$ coverages are matched between these two lines.

since $\mathrm{CO}$ is already thermalized and the population is at higher$\mathrm{J}$ transitions, which makes the lower-J lines optically thin and therefore low brightness temperatures. This is consistent with the non-detection of the $\mathrm{CO}$ lines toward the nucleus under the current noise level of $\sim 2-3 \mathrm{~K}$ at $3 \sigma$ upper limit (see Figure 3) and the detection of the $\mathrm{HCN}(1-0)$ line.

In summary, infrared pumping does not need to be invoked to realize a high $\mathrm{HCN}(1-0) / \mathrm{CO}(1-0)$ ratio of $>2$, but with infrared pumping, it is easier to realize. Since this is not conclusive only with the LVG calculations, we discuss the possibility of infrared pumping with other observational results in the next subsection.

\subsubsection{Possible Causes of High HCN Abundance}

Based on the radiative transfer calculations above, it is necessary to have a high $\mathrm{HCN}$ abundance to realize a high $\mathrm{HCN}(1-0) / \mathrm{CO}(1-0)$ ratio. There are several ways in which the $\mathrm{HCN}$ abundance may be enhanced. One possibility is due to high X-ray radiation from AGNs. Since X-ray radiation penetrates much deeper and denser regions than UV radiation, the chemical reactions exhibits different pathways compared with photodissociated region. The molecular gas region that is irradiated by X-ray is called X-ray-dominated region (XDR; Maloney et al. 1996), and some radiative transfer calculations of XDR succeeded to enhance the HCN abundance (Lepp \& Dargarno 1996; Lintott \& Viti 2006), but some do not (Meijerink \& Spaans 2005; Meijerink et al. 2007). A second possibility is energetic particles (i.e., cosmic rays) either from AGNs or radio jets. This possibility, however, reduces the $\mathrm{HCN}$ and $\mathrm{HNC}$ abundances, and increases the $\mathrm{HCO}^{+}$abundance (Bayet et al. 2010), which do not match with our results. Another possibility is the high temperature gas phase chemistry as recently suggested by Harada et al. (2010, 2013). This radiative transfer calculation includes the time-dependent interstellar chemical gas-phase reaction network, so that this calculation gives resultant molecular gas abundances under strong heating by X-ray irradiation. The calculation results show high HCN abundance, which matches with our observations. Furthermore, the HNC abundance increase is an order of magnitude less than that for the $\mathrm{HCN}$ abundance, and the $\mathrm{HCO}^{+}$abundance shows decrement under this model, which again matches with our results of non-detection for the $\mathrm{HCO}^{+}(1-0)$ and $\mathrm{HNC}(1-0)$ lines. Indeed, the $\mathrm{HCO}^{+}$vibrational absorption line at $12 \mu \mathrm{m}$ has not been detected toward the luminous infrared galaxies from which the HCN vibrational absorption line at $14 \mu \mathrm{m}$ has been detected (Imanishi et al. 2010). Since the $\mathrm{HCO}^{+}$ vibrational transition is located at a similar wavelength and has a similar Einstein coefficient as that of the $\mathrm{HCN}$ vibrational transition, non-detection of the $\mathrm{HCO}^{+}$absorption line suggests that the $\mathrm{HCN}$ abundance has enhanced compared to that of the $\mathrm{HCO}^{+}$, consistent with this model. In addition, high $\mathrm{HCN} / \mathrm{HNC}$ abundance ratios have been observed toward active star forming cores (Goldsmith et al. 1986; Schilke et al. 1992), and low ratios in starless cores (Hirota et al. 1998) in our Galaxy, which is again consistent with this model. Our results therefore strongly support the model presented in Harada et al. (2010, 2013).

On the other hand, the radiative transfer calculation results in Section 4.3 .2 cannot realize $\mathrm{HCN}(1-0) / \mathrm{CO}(3-2)$ above unity even under the HCN abundance enhanced conditions; in the $\mathrm{CO}(3-2)$ map (Figure 3), contours at the level of 3-4 $\sigma$, namely, 1.3-1.7 K, are at the nucleus, but no intensity peak has been observed as in the HCN map, if we consider the beam size. This suggests that $\mathrm{HCN}(1-0)$ is stronger than $\mathrm{CO}(3-2)$ at the 

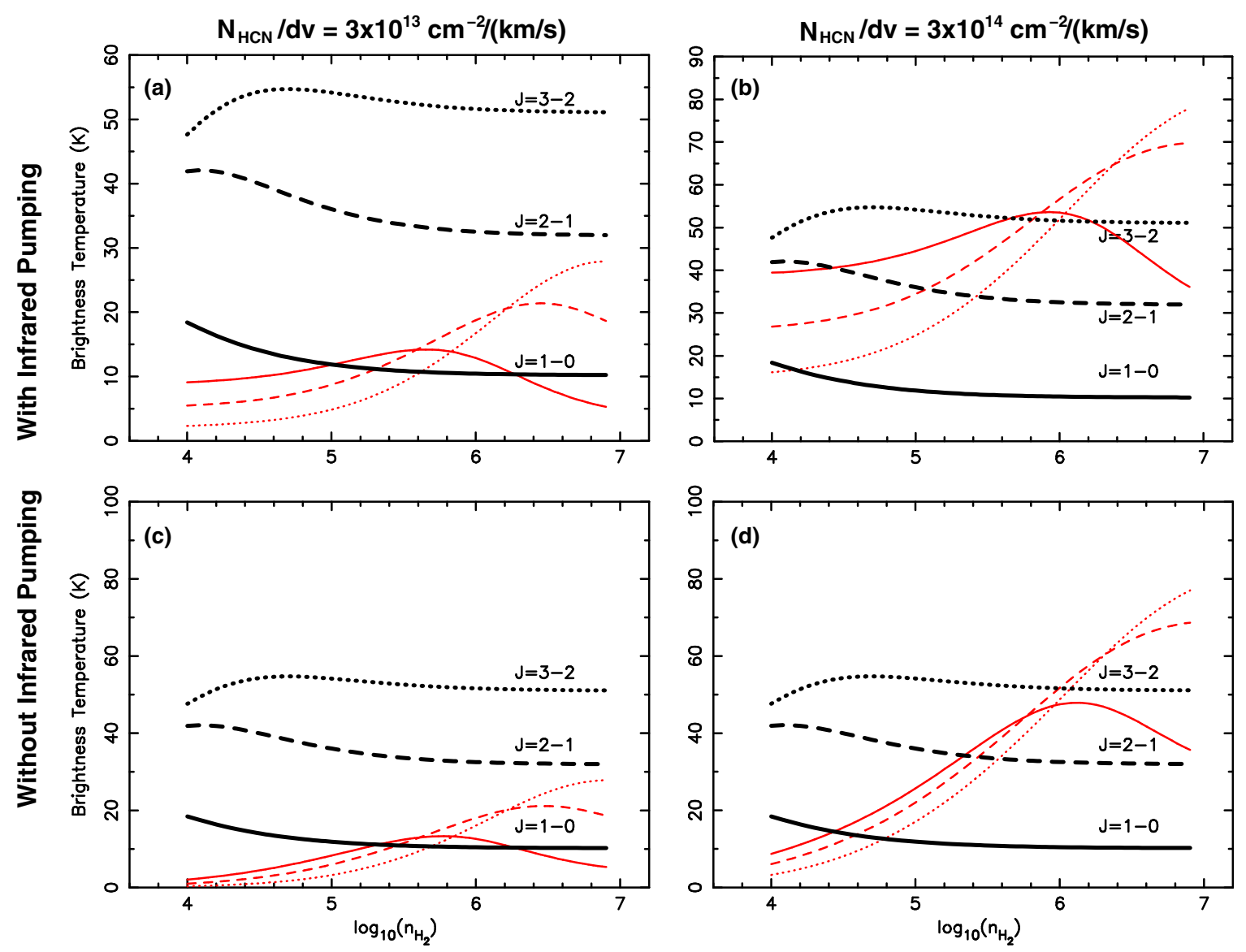

Figure 7. LVG calculation results with (upper row plots) and without (bottom row plots) IR pumping. We adopted the kinetic temperature of molecular gas as $100 \mathrm{~K}$. Plots are brightness temperatures (kelvin) of $\mathrm{CO}$ (black lines) and $\mathrm{HCN}$ (red lines) as a function of $\mathrm{H}_{2}$ number density $\left(\mathrm{cm}^{-3}\right.$ ). Solid, dashed, and dotted lines are the results for $J=1-0,2-1$, and 3-2 transitions, respectively. (a) Result with $N_{\mathrm{HCN}} / d v$ of $3 \times 10^{13} \mathrm{~cm}^{-2}\left(\mathrm{~km} \mathrm{~s}^{-1}\right)^{-1}$ and with IR pumping. (b) Result with $N_{\mathrm{HCN}} / d v$ of $3 \times 10^{14} \mathrm{~cm}^{-2}\left(\mathrm{~km} \mathrm{~s}^{-1}\right)^{-1}$ and with IR pumping. (c) Result with $N_{\mathrm{HCN}} / d v$ of $3 \times 10^{13} \mathrm{~cm}^{-2}\left(\mathrm{~km} \mathrm{~s}^{-1}\right)^{-1}$ but without IR pumping. (d) Result with $N_{\mathrm{HCN}} / d v$ of $3 \times 10^{14} \mathrm{~cm}^{-2}\left(\mathrm{~km} \mathrm{~s}^{-1}\right)^{-1}$ but without IR pumping.

nucleus, which is inconsistent with the calculation results in Figure 7. One possible mechanism to make the $\mathrm{HCN}(1-0)$ emission strong is weak $\mathrm{HCN}$ masing. The infrared pumping can invert the population at the $\mathrm{HCN}(1-0)$ transition and possible to produce the maser emission at this transition (Dinh-V-Trung \& Nguyen-Q-Rieu 2000). The HCN(1-0) masers have indeed been observed toward various carbon stars (Izumiura et al. 1987, 1995; Olofsson et al. 1993). Under this excitation condition, and if the HCN molecule is distributed as an edge-on torus or disk that is similar to the water maser disk in NGC 4258 (Miyoshi et al. 1995), it is possible to create $\mathrm{HCN}$ maser emission at the systemic velocity. This matches well with the strong $\mathrm{HCN}(1-0)$ nuclear emission only at the systemic velocity channel (Figures 3 and 4). A similar explanation has been discussed for the strong spike in the $\mathrm{HNC}(3-2)$ emission for the western nucleus of Arp 220 (Aalto et al. 2009), although our observation has not detected any HNC emission, probably due to the high temperature gas phase chemistry in our case, as mentioned above.

Our results therefore suggest that there is a compact dense molecular gas in front of the type 2 AGN in M51 that cannot be resolved in our spatial resolution of $\sim 1^{\prime \prime}(\sim 34 \mathrm{pc})$. This component can be the obscuring material for the type 2 nucleus of M51; assume this component has a size of $\sim 1$ pc (i.e., much smaller than the spatial resolution of our observations) along the line of sight and a density of $\sim 10^{6} \mathrm{~cm}^{-3}$ (i.e., consistent with the radiative transfer calculation results in Section 4.3.2), then the column density along the line of sight will be calculated as $\sim 3 \times 10^{24} \mathrm{~cm}^{-2}$. This value is consistent with the column density of $\sim 5.6 \times 10^{24} \mathrm{~cm}^{-2}$ for the absorption material in front of the nuclear X-ray source (i.e., AGN) of M51 (Fukazawa et al. 2001). It is highly required to observe HCN lines with $\ll 1^{\prime \prime}$ resolution toward M51 to directly image the obscuring material in front of the AGN, which may give us knowledge about the still-unknown obscuring and feeding mechanisms of AGNs.

\section{SUMMARY}

Our newly obtained $\sim 1^{\prime \prime}(\sim 34 \mathrm{pc})$ resolution $\mathrm{HCN}(1-0)$ image toward the Seyfert 2 nucleus of M51 (NGC 5194) revealed the following results.

1. The overall $\mathrm{HCN}(1-0)$ distribution and kinematics are similar to those of the CO lines. Since it is known that the kinematics derived from the $\mathrm{CO}$ lines indicates the jetentrained nature, this similarity indicates that the dense gas is also entrained by the jets and outflowing from the AGN.

2. $\mathrm{HCN}(1-0) / \mathrm{CO}(1-0)$ brightness temperature ratio is strongly enhanced along the jets with the value around unity, and the value is similar to that of the shocked gas in the outflows from young stellar objects in our Galaxy, suggesting that the HCN enhancement along the jets are highly related to shock.

3. A strong $\mathrm{HCN}(1-0)$ emission has been detected toward the nucleus only at the systemic velocity channel. No CO 
emission has been detected at this region and at this velocity, and therefore the ratio turned out to be more than 2 . To realize this high ratio, it is suggested from our radiative transfer model that weak HCN maser triggered by the infrared pumping with $\mathrm{HCN}$ abundance enhancement is the possible mechanism. The most plausible configuration for emitting the $\mathrm{HCN}$ maser is a rotating edge-on dense molecular gas disk or torus around the AGN, similar to the water maser disks in other Seyfert 2 galaxies. If this disk or torus has the size of $\sim 1 \mathrm{pc}$ and the density of $\sim 10^{6} \mathrm{~cm}^{-2}$, then it is possible to explain the nuclear obscuration for this type 2 AGN in M51.

4. Cause of the high HCN abundance can be due to high temperature gas phase chemistry. Non-detection of the $\mathrm{HCO}^{+}(1-0)$ and $\mathrm{HNC}(1-0)$ lines in our observations also support this model.

We would like to thank the IRAM and SMA staff for supporting our observations. We also thank Dr. T. Umemoto for the permission to use the molecular line spectra of L1157, and Dr. F. Egusa for providing us the flux information of the nucleus of M51 from the AKARI Infrared Camera data. We are also grateful to the anonymous referee for very helpful comments and discussion. This collaboration was partly supported by the French-Taiwanese Hubert Curien Program, ORCHID. S.M. is supported by the National Science Council (NSC) and the Ministry of Science and Technology (MoST) of Taiwan, NSC 97-2112-M-001-021-MY3, NSC 100-2112-M-001-006-MY3, and MoST 103-2112-M-001-032-MY3. D.V.T. is funded by the project "Development of problem-solving environments for computational science based on resource sharing highperformance computers at Vietnam Academy of Science and Technology."

\section{REFERENCES}

Aalto, S., Booth, R. S., Black, J. H., \& Johansson, L. E. B. 1995, A\&A, 300,369

Aalto, S., Garcia-Burillo, S., Muller, S., et al. 2012, A\&A, 537, A44

Aalto, S., Wilner, D., Spaans, M., et al. 2009, A\&A, 493, 481

Antonucci, R. R. J. 1993, ARA\&A, 31, 473

Bayet, E., Hartquist, T. W., Viti, S., Williams, D. A., \& Bell, T. A. 2010, A\&A, 521, A16

Bradley, L. D., Kaiser, M. E., \& Baan, W. A. 2004, ApJ, 603, 463

Brouillet, N., Muller, S., Herpin, F., Braine, J., \& Jacq, T. 2005, A\&A, 429,153

Carrol, T. J., \& Goldsmith, P. F. 1981, ApJ, 245, 891

Combes, F., \& Becquaert, J.-F. 1997, A\&A, 326, 554

Combes, F., García-Burillo, S., Casasola, V., et al. 2013, A\&A, 558, A124

Crane, P. C., \& van der Hulst, J. M. 1992, AJ, 103, 1146

Davies, R. I., Maciejewski, W., Hicks, E. K. S., et al. 2009, ApJ, 702,114

Deguchi, S., \& Uyemura, M. 1984, ApJ, 285, 153

Dinh-V-Trung, \& Nguyen-Q-Rieu 2000, A\&A, 361, 601

Dumouchel, F., Faure, A., \& Lique, F. 2010, MNRAS, 406, 2488

Egusa, F., Wada, T., Sakon, I., et al. 2013, ApJ, 778, 1

Farrah, D., Bernard-Salas, J., Spoon, H. W. W., et al. 2007, ApJ, 667, 149

Ford, H. C., Crane, P. C., Jacoby, G. H., Lawrie, D. G., \& van der Hulst, J. M. 1985, ApJ, 293, 132

Fukazawa, Y., Iyomoto, N., Kubota, A., Matsumoto, Y., \& Makishima, K. 2001, A\&A, 374, 73

Gao, Y., \& Solomon, P. M. 2004, ApJ, 606, 271

García-Burillo, S., Combes, F., Usero, A., et al. 2014, A\&A, 567, A125
Goldsmith, P. F., Irvine, W. M., Hjalmarson, A., \& Ellder, J. 1986, ApJ, 310,383

Hagiwara, Y., Henkel, C., Menten, K. M., \& Nakai, N. 2001, ApJL, 560, L37

Harada, N., Herbst, E., \& Wakelam, V. 2010, ApJ, 721, 1570

Harada, N., Thompson, T. A., \& Herbst, E. 2013, ApJ, 765, 108

Harris, G. J., Tennyson, J., Kaminsky, B. M., Pavlenko, Ya. V., \& Jones, H. R. A. 2006, MNRAS, 367, 400

Helfer, T. T., \& Blitz, L. 1993, ApJ, 419, 86

Helfer, T. T., \& Blitz, L. 1997, ApJ, 478, 233

Hicks, E. K. S., Davies, R. I., Malkan, M. A., et al. 2009, ApJ, 696, 448

Hirota, T., Yamamoto, S., Mikami, H., \& Ohishi, M. 1998, ApJ, 503, 717

Ho, L. C., Filippenko, A. V., \& Sargent, W. L. W. 1997, ApJS, 112, 315

Ho, P. T. P., Moran, J. M., \& Lo, F. 2004, ApJ, 616, L1

Hsieh, P.-Y., Matsushita., S., Lim, J., Kohno, K., \& Sawada-Satoh, S. 2008, ApJ, 683,70

Imanishi, M., \& Nakanishi, K. 2013, AJ, 146, 91

Imanishi, M., Nakanishi, K., Yamada, M., Tamura, Y., \& Kohno, K. 2010, PASJ, 62, 201

Irvine, W. M., Goldsmith, P. F., \& Hjalmarson, A. 1987, in Interstellar Process, ed. D. J. Hollenbach \& H. A. Thronson (Dordrecht: Reidel), 585

Izumiura, H., Ukita, N., Kawabe, R., et al. 1987, ApJL, 323, L81

Izumiura, H., Ukita, N., \& Tsuji, T. 1995, ApJ, 440, 728

Jackson, J. M., Heyer, M. H., Paglione, T. A. D., \& Bolatto, A. D. 1996, ApJL, 456, L91

Jackson, J. M., Paglione, T. A. D., Ishizuki, S., \& Rieu, N. Q. 1993, ApJL, 418, L13

Jacquinet-Husson, N., Crepeau, L., Armante, R., et al. 2011, JQSRT, 112, 2395

Jaffe, W., Meisenheimer, K., \& Röttgering, H. J. A. 2004, Natur, 429, 47

Kohno, K., Kawabe, R., Tosaki, T., \& Okumura, S. K. 1996, ApJL, 461, L29

Kohno, K., Matsushita, S., Vila-Vilaró, B., et al. 2001, in ASP Conf. Ser. 249, The Central Kiloparsec of Starbursts and AGN: The La Palma Connection, ed. J. H. Knapen et al. (San Francisco, CA: ASP), 672

Kohno, K., Muraoka, K., Hatsukade, B., et al. 2008a, in Far-Infrared Workshop 2007, ed. C. Kramer, S. Aalto, \& R. Simon (EAS Publication Ser., Vol. 31; Les Ulis, France; EDP Sciences), 65

Kohno, K., Nakanishi, K., Tosaki, T., et al. 2008b, Ap\&SS, 313, 279

Krips, M., Martín, S., Eckart, A., et al. 2011, ApJ, 736, 37

Krips, M., Neri, R., García-Burillo, S., et al. 2007, A\&A, 468, L63

Lahuis, F., Spoon, H. W. W., Tielens, A. G. G. M., et al. 2007, ApJ, 659, 296

Lepp, S., \& Dalgarno, A. 1996, A\&A, 306, L21

Lintott, C., \& Viti, S. 2006, ApJL, 646, L37

Maloney, P. R., Hollenbach, D. J., \& Tielens, A. G. G. M. 1996, ApJ, 466, 561

Matsushita, S., Kawabe, R., Kohno, K., Tosaki, T., \& Vila-Vilaró, B. 2010, PASJ, 62, 409

Matsushita, S., Kohno, K., Vila-Vilaró, B., Tosaki, T., \& Kawabe, R. 1998, ApJ, 495, 267

Matsushita, S., Muller, S., \& Lim, J. 2007, A\&A, 468, L49

Matsushita, S., Sakamoto, K., Kuo, C.-Y., et al. 2004, ApJL, 616, L55

Meijerink, R., \& Spaans, M. 2005, A\&A, 436, 397

Meijerink, R., Spaans, M., \& Israel, F. P. 2007, A\&A, 461, 793

Miyoshi, M., Moran, J., Herrnstein, J., et al. 1995, Natur, 373, 127

Morris, M. 1975, ApJ, 197, 603

Müller Sánchez, F., Davies, R. I., Genzel, R., et al. 2009, ApJ, 691, 749

Olofsson, H., Eriksson, K., Gustafsson, B., \& Carlstrom, U. 1993, ApJS, 87,305

Sakamoto, K., Aalto, S., Evans, A. S., Wiedner, M. C., \& Wilner, D. J. 2010, ApJL, 725, L228

Salter, C. J., Ghosh, T., Catinella, B., et al. 2008, AJ, 136, 389

Sani, E., Davies, R. I., Sternberg, A., et al. 2012, MNRAS, 424, 1963

Schilke, P., Walmsley, C. M., Pineau Des Forets, G., et al. 1992, A\&A, 256, 595

Scoville, N. Z., Yun, M. S., Armus, L., \& Ford, H. 1998, ApJL, 493, L63

Shetty, R., Vogel, S. N., Ostriker, E. C., \& Teuben, P. J. 2007, ApJ, 665, 1138

Solomon, P. M., Downes, D., \& Radford, S. J. E. 1992, ApJL, 387, L55

Sorai, K., Nakai, N., Kuno, N., \& Nishiyama, K. 2002, PASJ, 54, 179

Takáts, K., \& Vinkó, J. 2006, MNRAS, 372, 1735

Tully, R. B. 1988, Nearby Galaxies Catalog (Cambridge: Cambridge Univ. Press)

Umemoto, T., Iwata, T., Fukui, Y., et al. 1992, ApJL, 392, L83

Van der Tak, F. F. S., Black, J. H., Schöier, F. L., Jansen, D. J., \& van Dishoeck, E. F. 2007, A\&A, 468, 627

Wright, M. C. H., Plambeck, R. L., \& Wilner, D. J. 1996, ApJ, 469, 216 